\title{
Sleep disturbances and suicide risk: A review of the literature
}

\author{
Rebecca A Bernert \\ Thomas E Joiner
}

Department of Psychology, Florida State University, Tallahassee, FL, USA
Correspondence:Thomas Joiner

Department of Psychology, Florida State University, Tallahassee, Florida 32306- 1270

Tel + I 850644 I454

$\mathrm{Fax}+\mid 8506457518$

Email joiner@psy.fsu.edu

\begin{abstract}
A growing body of research indicates that sleep disturbances are associated with suicidal ideation and behaviors. This article (1) provides a critical review of the extant literature on sleep and suicidality and (2) addresses shared underlying neurobiological factors, biological and social zeitgebers, treatment implications, and future directions for research. Findings indicate that suicidal ideation and behaviors are closely associated with sleep complaints, and in some cases, this association exists above and beyond depression. Several cross-sectional investigations indicate a unique association between nightmares and suicidal ideation, whereas the relationship between insomnia and suicidality requires further study. Underlying neurobiological factors may, in part, account for the relationship between sleep and suicide. Serotonergic neurotransmission appears to play a critical role in both sleep and suicide. Finally, it remains unclear whether or not sleep-oriented interventions may reduce risk for suicidal behaviors. Unlike other suicide risk factors, sleep complaints may be particularly amenable to treatment. As a warning sign, disturbances in sleep may thus be especially useful to research and may serve as an important clinical target for future suicide intervention efforts.
\end{abstract}

Keywords: suicidality, sleep, nightmares, suicide risk factors

\section{Introduction}

Suicide is a leading cause of death. Approximately 30,000 people die by suicide each year in the United States alone (Murphy 2000). Suicide kills more Americans annually than homicides, and although rates vary substantially by age, suicide ranks as the 11 th most common cause of death. Attempted suicides are believed to far exceed this number. It is estimated that 10 to 25 nonlethal suicide attempts occur for every completed suicide (Maris 2002). Moreover, such attempts are responsible for more than 400,000 emergency room visits annually (Doshi et al 2005). Taken together, suicidal behaviors represent a complex, yet potentially preventable public health problem, with far-reaching personal and societal consequences. Improvements in the identification of risk factors for suicidal behaviors thus ultimately enhance our ability to intervene and prevent death by suicide.

Acute and chronic suicide risk is associated with social, psychological, and biological variables (Lewinsohn et al 1996; Mann et al 2001; Rowe et al 2006), and such factors are often further divided into precipitating and predisposing causes (Mann 2002). One growing area of research includes the study of sleep complaints and suicidality. Increasing evidence suggests that disturbances in sleep are associated with an elevated risk for suicidal behaviors. Both sleep disorders and general sleep complaints appear to be linked to greater levels of suicidal ideation and depression, as well as both attempted and completed suicide (Krakow et al 2000; Agargun et al 1997a; Fawcett et al 1990). In consideration of these findings, sleep problems and more specifically, significant changes in sleep, are now listed among the top 10 warning signs of suicide from the Substance Abuse and Mental Health Services Administration (SAMSHA) (National Mental Health Information Center 2005). 


\section{Clinical and epidemiological investigations of self-reported sleep disturbances and suicidal behaviors Poor sleep quality, insomnia, and hypersomnia}

Fawcett and colleagues conducted one of the first studies to prospectively examine sleep, depression, and suicide (Fawcett et al 1990). In a group of depressed patients, symptoms of global insomnia were more severe among those who completed suicide within a 13-month period. This finding suggested that insomnia may be considered a clinical indicator of acute suicidal risk, perhaps particularly when it appears in the midst of a depressive episode. Agargun et al (1997a) demonstrated a similar link between suicidality, depression, and sleep complaints. Depressed subjects suffering from either hypersomnia or insomnia showed significantly higher scores on measures of suicidality. In a separate study, these authors also examined self-reported sleep quality among depressed patients (Agargun et al 1997b). Subjective sleep quality was significantly more disturbed among suicidal versus nonsuicidal patients.

An association between poor sleep quality and completed suicide has been prospectively studied in several community samples of men and women. Among a large group of elderly participants, for example, poor self-reported sleep quality was linked to suicide within 10 years. Although depression showed the strongest link with suicide, poor sleep quality increased the risk for suicide by 34\% (Turvey et al 2002). A recent investigation conducted in Japan demonstrated similar findings. Fujino et al (2005) showed that, among 13,259 middle-aged adults, only difficulty maintaining sleep at baseline, compared to other sleep disturbances (eg, difficulty initiating sleep, nonrestorative sleep), significantly predicted death by suicide 14 years later. In both of these studies, depression was not accounted for when examining the association between sleep and completed suicide. Such information would elucidate whether sleep disturbance stands alone as a risk factor for completed suicide or, conversely, whether such sleep complaints simply vary with increased depressive symptoms.

\section{Nightmares}

Similar to insomnia symptoms, nightmares are more common among suicidal versus nonsuicidal individuals with major depression. Research indicates that depressed patients with self-reported repetitive and frightening dreams are more likely to be classified as suicidal, compared to those without frequent nightmares (Agargun et al 1998). A similar relationship recently emerged in a prospective, population-based study conducted in Finland. Tanskanen et al revealed an association between nightmare frequency at baseline and completed suicides at follow-up 14 years later (Tanskanen et al 2001). Compared to subjects reporting no nightmares, those reporting occasional nightmares were 57\% more likely to die by suicide. Among those with frequent nightmares, the risk for suicide increased dramatically; those endorsing frequent nightmares were $105 \%$ more likely to die by suicide compared to those reporting no frightening dreams.

Bernert and colleagues (2005) investigated the frequency and severity of nightmare symptoms, depression, and suicidality among 176 clinical outpatients using several validated symptom inventories. Results indicated that nightmares predicted elevated suicidal ideation, and this effect was independent of depression. Although this relationship emerged as a nonsignificant trend $(p=0.06)$, these findings suggest that nightmares may constitute a unique risk factor for elevated suicidality. More recently, Agargun and colleagues (2007) examined nightmare frequency, insomnia symptoms, and suicide attempt status among depressed patients with and without melancholic features. Depressed patients with melancholic features $(\mathrm{N}=100)$ were compared to depressed patients without these features $(\mathrm{N}=49)$. Participants were categorized further as having a history or no history of suicide attempts. Results revealed that melancholic patients with a history of suicide attempts showed higher rates of nightmares and insomnia symptoms compared to melancholic patients without a history of attempts. This study did not assess sleep variables using objective sleep tests; however, it is perhaps the first investigation to examine melancholic depression, suicidality, and sleep disturbances. The authors theorized that feeling worse in the morning as opposed to later in the day, a hallmark symptom of melancholic depression, may be associated with dream content, more negative affect, and in this way, greater risk for suicidality.

\section{EEG sleep studies, sleep-disordered breathing, and suicidal behaviors}

Sleep abnormalities in depression and suicide have also been investigated using objective measurements of sleep (Sabo et al 1991). In a retrospective analysis of sleep architecture, Sabo et al compared depressed patients with and without a history of suicide attempts. Electroencephalographic (EEG) sleep studies revealed that those with a history of suicide attempts showed a lower sleep efficiency, a longer sleep latency, and fewer late-night delta counts. More recently, Agargun and 
Cartwright investigated the relationship between rapid eye movement (REM) sleep, dream variables, and suicidality in depression (Agargun and Cartwright 2003). Compared with nonsuicidal subjects, suicidal patients averaged a shorter REM sleep latency, a higher REM percentage, and a more negative dream-like quality of REM. As with the reports reviewed above, these studies failed to investigate the link between sleep and suicide after accounting for the variance associated with depression.

Apart from nightmares and insomnia, other sleep complaints, such as sleep-disordered breathing, have been researched even less frequently with regard to suicidality and depression. To our knowledge, only one study has investigated the relationship between sleep-disordered breathing, suicidal ideation, and depressive symptoms. Krakow and colleagues examined subjective sleep disturbances in 153 female sexual assault survivors with posttraumatic stress disorder (Krakow et al 2000). Each woman completed various questionnaires assessing sleep quality, depressive symptoms, and suicidality. Participants were originally recruited for a nightmare-treatment program, which may have inflated the prevalence of other co-occurring sleep complaints. Nonetheless, results indicated that women who experienced a potential sleep breathing disorder also suffered significantly greater levels of depression and suicidal ideation. Problematic breathing during sleep is often associated with frequent arousals throughout the sleep period. These authors speculated that physiological sleep fragmentation might contribute to "emotional exhaustion," which has been reported in patients exhibiting suicidal behavior.

\section{Sleep disturbances, depression, mood regulation, and suicidal behaviors}

Despite the connection observed between sleep and suicide, it remains relatively unclear how these two constructs relate to one another after controlling for depression. In several reports, the association between sleep and suicide did not appear to be entirely independent of depressive symptoms (Krakow et al 2000), while in other investigations, suicidality and sleep were only assessed among depressed individuals (Sabo et al 1991; Agargun et al 1997a, 1997b). Although sleep disturbances are linked to suicidality, this relationship may largely be explained by higher depression ratings. Only a few studies have examined the connection between suicide and sleep complaints beyond that explained by depression. First, Keshavan et al (1994) examined REM sleep in psychotic patients with and without a history of suicide attempts or ideation. Patients with a history of suicidal behavior showed more REM activity, and REM sleep parameters were not correlated with depression scores. When depression ratings were covaried out, differences in sleep remained. Next, in a study by Smith, Perlis, and Haythornthwaite (2004), chronic pain patients $(\mathrm{N}=51)$ were classified as "ideators" or "non-ideators" based on their response to Item 9 on the Beck Depression Inventory (BDI). Those who reported sleep-onset insomnia symptoms were significantly more likely to report suicidal ideation, and this effect was found to be independent of depression severity. In 2005, Bernert and colleagues examined self-reported sleep complaints and suicidality among 176 psychiatric outpatients. After controlling for depressive symptoms and sex, the association between sleep (nightmares specifically) and suicidality remained significant, whereas insomnia and sleep-disordered breathing symptoms and suicidality did not. These results converge somewhat with findings from adolescent investigations. Liu and colleagues (2004) showed that nightmares were strongly associated with suicidal ideation, even after controlling for mood symptoms; insomnia symptoms did not remain significantly associated with increased ideation after controlling for depression. In contrast to these results, however, another study (Roberts et al 2001) showed that insomnia was a predictor of elevated suicidal ideation with depression as a covariate. Although these data suggest convergence with regard to nightmares as a unique predictor of ideation, the association between insomnia and suicidality remains mixed.

Mood regulation factors may play a primary role in the relationship between sleep and suicide. Sleep may, for example, fail to provide an emotional refuge for distressed individuals. Frequent nightmares may exacerbate feelings of ineffectiveness, and poor sleep quality may disrupt within-sleep mood regulation processes. This explanation is supported by research conducted among depressed samples. For example, Cartwright et al (1998) examined a moodregulatory hypothesis of dreaming among depressed women over a one-year period. Women reporting more affectively negative dreams early in the night, yet fewer negative dreams at the end of the sleep period, were more likely to achieve remission within one year; in contrast, those reporting few negative dreams at the beginning of the night, yet more negative dreams at the end of the sleep period, were less likely to achieve remission a year later. These findings suggest that the ability to regulate one's mood during sleep has a significant and long-term impact on psychopathology. More recently, Agargun and Cartwright (2003) also demonstrated that suicidal patients showed a more negative dream-like 
quality of REM. Although both of these studies exclusively examined depressed individuals, such reports suggest that dream content and within-sleep mood regulation processes may play a significant role in the association between sleep and suicidality. Future research is needed to determine if: (1) the relationship between mood dysregulation and suicidal behaviors is confined to the sleep period only, or (2) wakingstate mood regulation factors also predict elevated suicidal ideation and behaviors. Such studies may, for example, clarify whether or not mood regulation presents risk strictly as a neurological or neurobiological process, or whether it also serves as a risk variable in the form of more broadlydefined, voluntary behaviors occurring during waking states (eg, increased emotional lability). Please see Table 1 for a selected list of all reports examining sleep and suicidality.

\section{Shared neurobiological factors}

The association between sleep disturbances and suicidal behavior suggests that these constructs may also share a common neurobiological basis. Serotonin (5-HT) appears to play a significant role in suicide and in the regulation of sleep. Serotonergic neurotransmission is involved in promoting and modulating behavioral states. Specifically, the release of serotonin appears to be highest during waking states, reduced during slow wave sleep, and lowest during REM sleep (Ursin 2002). Although the link between serotonin and sleep is more complicated than once originally thought, recent research suggests that serotonin release during waking states drives the homeostatic regulation of slow wave sleep (Jouvet 1999). Serotonergic dysfunction, particularly a reduction in the synthesis of serotonin, is believed to promote wakefulness (Ursin 2002; Adrien 2002). Interestingly, recent animal research indicates that the sensitivity of serotonin functioning is influenced by sleep loss itself. In rats, Roman et al (2005) demonstrated that sleep restriction (4 hours) for 8 days resulted in a desensitized 5-HT 1A receptor system, and this effect persisted despite unlimited time for sleep recovery. This study may have important implications for the pathogenesis of other disorders or behaviors associated with serotonergic dysfunction, such as suicidality.

As indicated earlier, serotonergic dysregulation appears to play an important role in conferring risk for suicide. Low cerebrospinal fluid (CSF) concentrations of 5-hydroxyindoleactic acid (5-HIAA) have been consistently observed in patients with a history of suicide. A number of studies have now documented a link between suicidal behavior and low 5-HIAA CSF concentrations, and this connection appears to cut across psychiatric conditions (Asberg 1997). Such studies also indicate that low levels of 5-HIAA CSF among suicide attempters predict future attempts. Those who have completed suicide, compared to controls, also show fewer serotonin transporter sites, more postsynaptic 5HT1A and 5HT2A receptors, and a higher number of less functional neurons (Maris 2002). A history of suicide attempts has additionally been linked to specific genotypes. According to a recent meta-analysis, psychiatric patients who possess the short allele of the 5-HTTLPR polymorphism show an increased risk for suicide (Lin and Tsai 2004).

Future research is warranted to elucidate shared etiological factors for suicide and sleep disturbances. Pharmacological treatments for depression will be an important factor to consider in designing such studies. In general, antidepressants appear to reduce risk for completed suicide, but questions remain as to whether or not such treatments may increase suicidality in some individuals (Healy and Whitaker 2003; Gibbons et al 2005). Research suggests that discontinuation of tricyclic (TCA) and selective serotonin reuptake inhibitor (SSRI) antidepressants confer up to a 5-fold increased risk for suicidal behaviors (Yerevanian et al 2004). Even so, the role of sleep in these situations remains relatively unclear. Active antidepressant treatment appears to induce specific changes in sleep architecture, whereas antidepressant discontinuation also results in sleep disturbances (Dominguez and Goodnik 1995; Warner et al 2006).

SSRIs have garnered considerable research attention in this regard. Sleep problems associated with SSRI treatment may be explained, in part, at the neurobiological level. Rapid Eye Movement (REM) sleep is inhibited by serotonergic and noradrenergic neurons (Siegel 1990; Leubkle et al 1992), and SSRIs increase the availability of both serotonin and noradrenaline in the brain. In this way, SSRI treatment is associated with REM suppression, and this effect has been observed among normal controls as well as depressed patients (Sharpley and Cowen 1995; Vogel et al 1990; Pace-Schott et al 2001). SSRI treatment is also tied to overall decreases in sleep efficiency (ie, percentage of time asleep of time spent in bed) among depressed participants (Trivedi 1999); however, the effects of antidepressants on sleep vary considerably by person, dose, and medication type (for review, see Mayers and Baldwin 2005). Taken together, history of antidepressant treatment may be an important variable to assess when examining sleep, serotonin, and risk for suicide.

\section{Biological and social zeitgebers}

Sleep is a complex behavioral phenomenon, driven by circadian and homeostatic sleep factors (Saper et al 2001). 


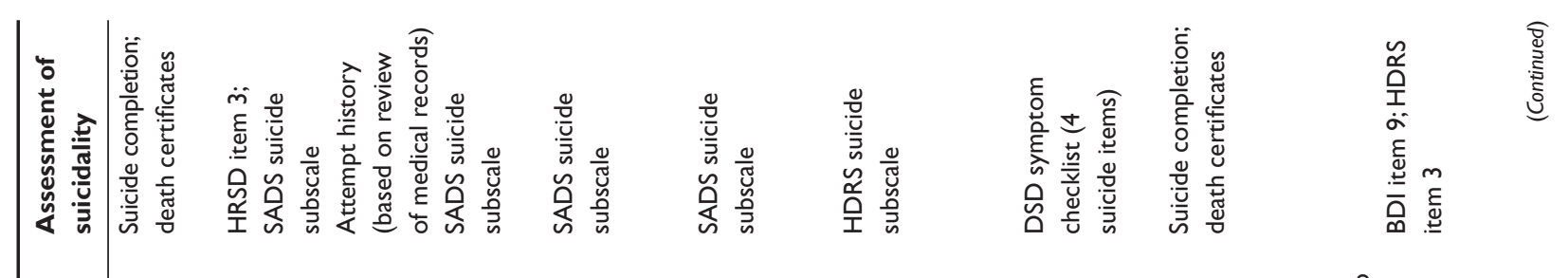

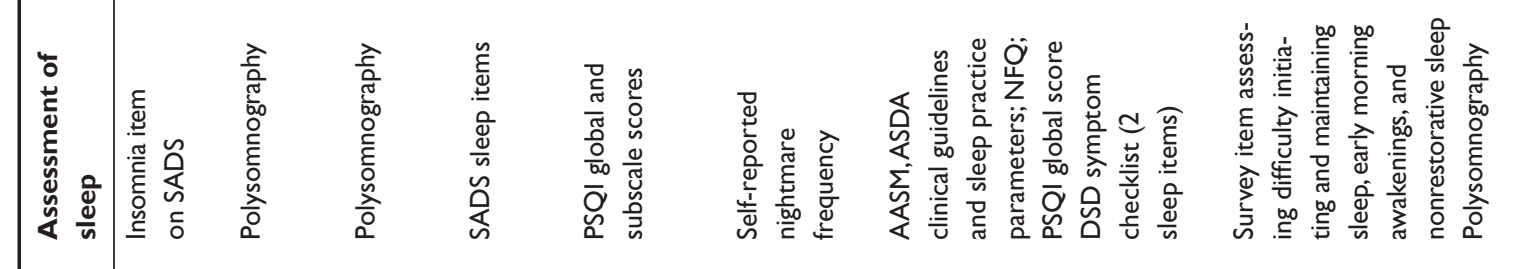

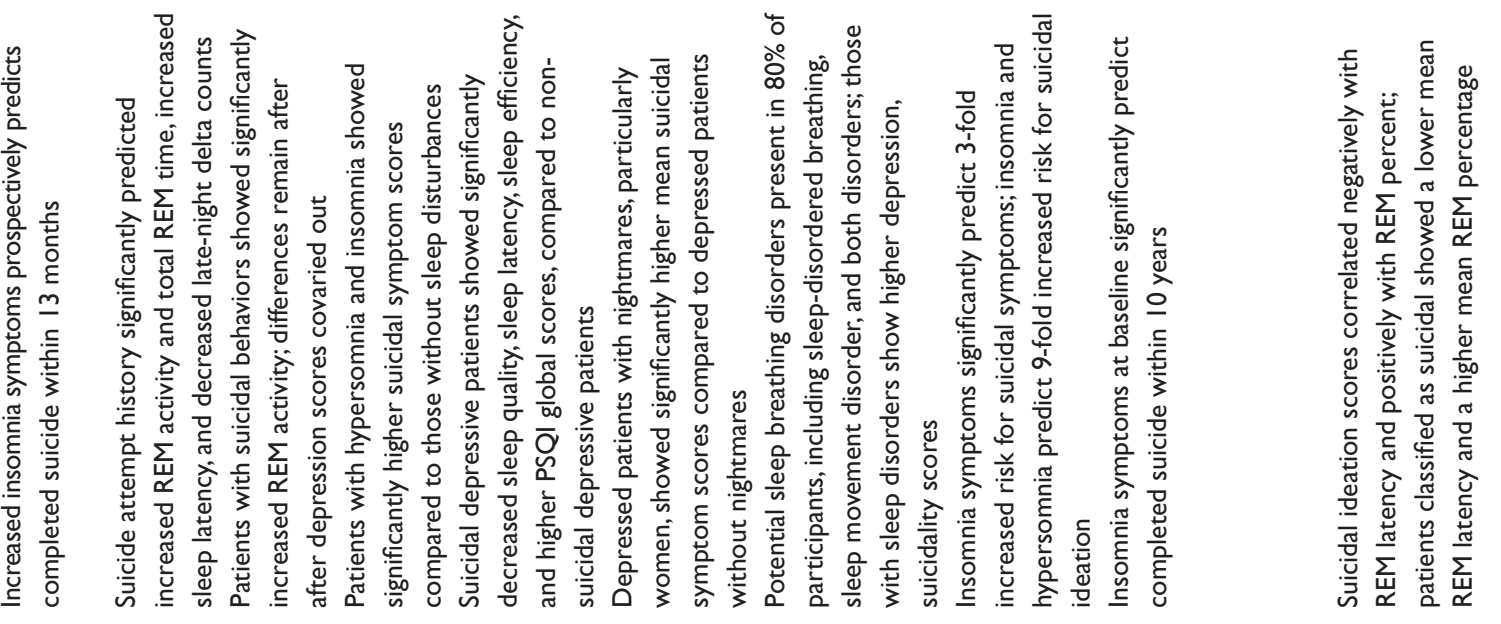
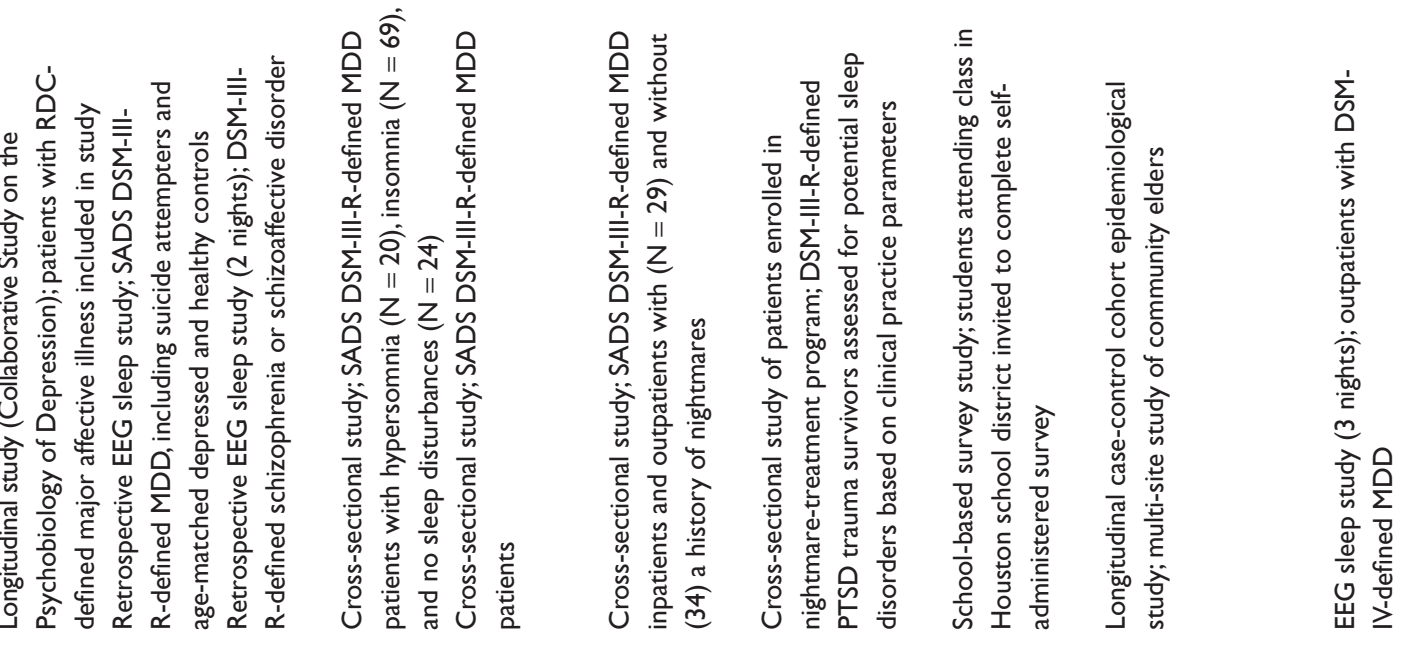

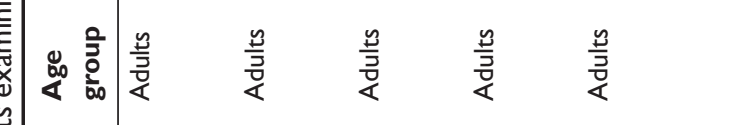

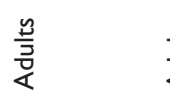

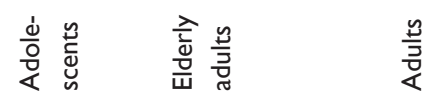

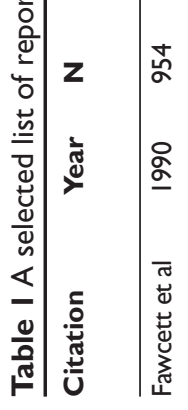

q $q \stackrel{m}{=} \bar{q}$

m

శ్

娄

$\stackrel{2}{2}$

$\overline{\mathrm{c}}$

ธิ่

ڤ̊

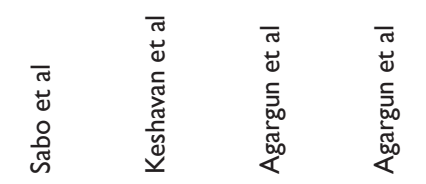

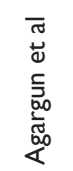

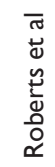

Ũ. 


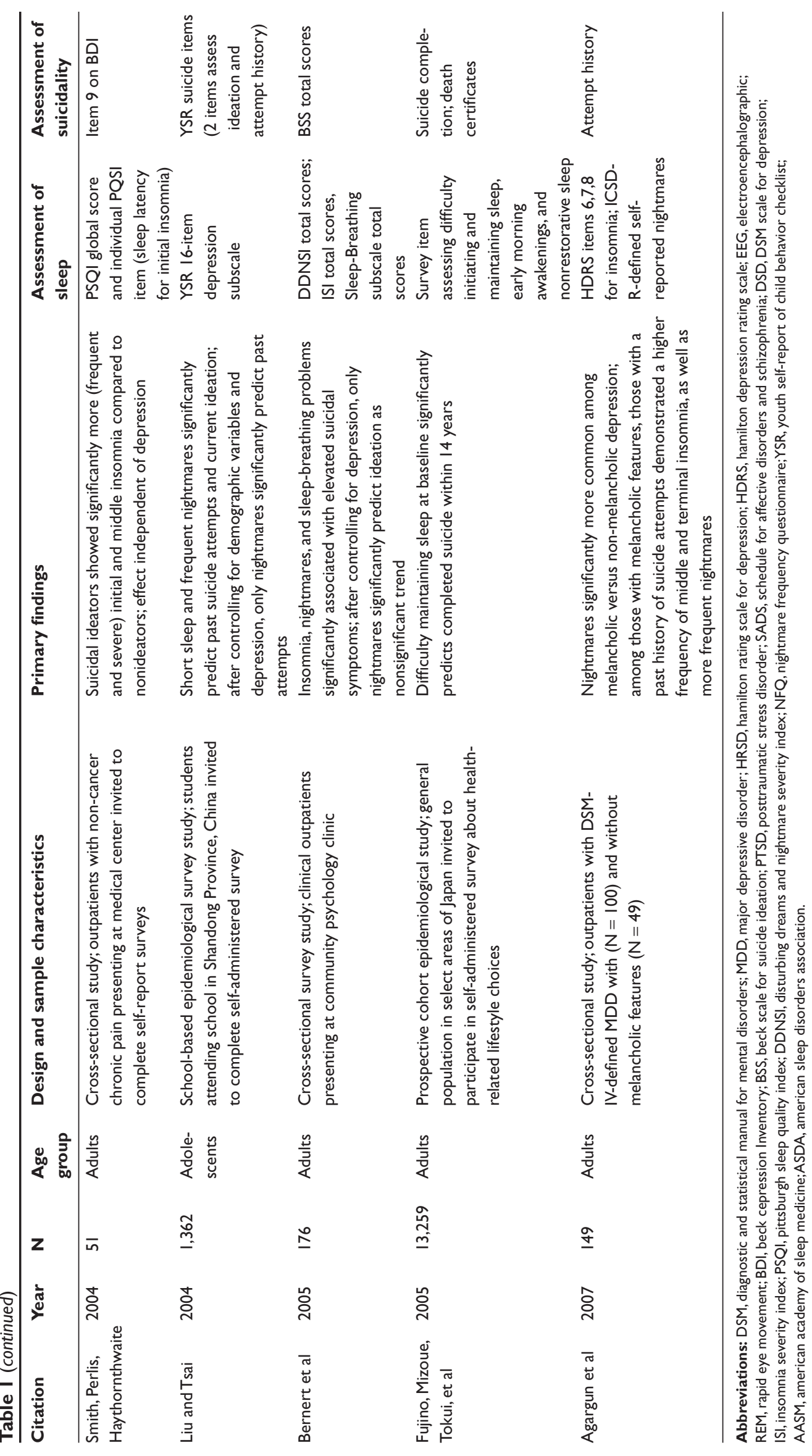


Zeitgebers are defined as external time cues that regulate and synchronize our internal body clock (DeCoursey 2004). Sleep and suicidal behaviors may be influenced by various time cues, which thus far, have received little attention in research. A diurnal variation in the timing of self-injurious behaviors and completed suicide is supported by several reports, although studies have demonstrated conflicting results.

Blenkiron et al (2000) prospectively assessed 158 patients presenting at a hospital referred for psychiatric assessment due to deliberate self harm. The time of day was recorded for each incident, and patients were asked to complete the Beck Suicide Intent Scale. The authors classified these deliberate self-harm incidents as "suicide attempts," and concluded that the frequency of these acts were highest in the evening (2200-2400) and lowest in the early morning hours (0200-0800). A significant relationship did not emerge for time of day and intent to die; however, those with acts earlier in the day were more likely to be admitted for inpatient medical services ( $70 \%$ vs. $46 \%$ ). This suggests that suicidal intent does not vary according to the time of day, although behaviors early in the morning had an increased likelihood of inpatient medical hospitalization. Based on these results, the authors therefore concluded that the severity of deliberate self-harm (though not intent to die, according to the Beck Suicide Intent Scale) appeared to vary according to the time of day. Those engaging in deliberate self-harm in the morning were significantly more likely to be older than those engaging in deliberate self-harm in the evening. In a separate study, ambulance report records were retrospectively reviewed for a 7-year period in Tokyo to examine time-of-day and documented suicide attempts. Results indicated that suicide attempts showed a peak earlier in the evening (1800) compared to the morning (Motahashi 1990).

Preti and Miotto (2001) examined diurnal variation in the timing of completed suicides. These authors examined 11,043 males and 3,700 females who died by suicide in Italy over a 4-year period (1994-1997). Among both genders, a significant time-dependent effect was observed in the distribution of suicides. Suicides peaked in the late morning (0800-1100), whereas rates were at their lowest in the late evening hours (2300-0300). When this relationship was examined by age, a bimodal distribution was observed for young and older adults. Those aged 65 and older showed a clear peak in the early morning (0800-1100), whereas young adults (aged 14-24) showed a peak in the late afternoon (1600-1900). Those aged 25-44 showed a less pronounced peak in the middle of the day (1100-1500). Van Houwelingen and Beersma (2001) found opposing findings when examining diurnal variation in train suicide rates among 2,830 victims in the Netherlands. Results indicated a 10-fold increased rate in the evening (2200-2400) compared to the early morning hours (0200-0600).

In summary of the above findings, suicide attempts and deliberate self-harm incidents appear to peak in the evening hours across age groups; however, this peak in frequency does not appear to be related to suicidal intent. Those engaging in deliberate self-harm in the morning are more likely to be older in age. For completed suicides, although the lowest rates of suicide tend to occur in the early morning hours, suicide peaks are reported for both the morning and evening. Conflicting results may be explained by the method of suicide, which, in one case, was restricted to train suicides. The distribution of suicides may also vary according to age, and studies differed in the extent to which they accounted for this variable. Additional research is needed to clarify whether suicidal behaviors are, indeed, a function of clock time, and if so, whether this relationship is associated with sleep, the light-dark cycle, and dysregulated mood states. Alternatively, diurnal variation in suicidal behaviors may also be explained by social factors, which may gate the opportunity for deliberate self-harm. For example, Blenkiron et al (2000) showed a bimodal peak in frequency for deliberate self-harm among older and younger adults (ie, deliberate self-harm among older adults peaked in the morning, whereas self-harm among younger adults peaked in the afternoon). It was theorized that this difference in timing might be explained by age-related differences in daytime schedules and social activities. Specifically, older individuals may be more likely to be alone in the morning, whereas adolescents, due to school schedules, may be more likely to be alone in the afternoon.

The study of chronobiological factors in the relationship between sleep and suicidal behaviors remains a largely unexplored, yet fruitful area of research, and is one which has been suggested elsewhere (Agargun and Besiroglu 2005). In studying time-related risk factors, additional research is needed, particularly studies that better define the severity of suicidal behaviors; it will be important for such studies to carefully distinguish suicide attempts and deliberate self-harm with an intent to die from self harm behaviors without suicidal intent. Investigation of the timing of sleep and suicidal acts may inform risk assessment procedures, emergency responding and surveillance, as well as treatment.

\section{Treatment implications and future directions}

Empirically-validated frameworks exist for routinizing suicide risk assessment procedures (Joiner et al 1999). 
Decision tree rules and symptom checklists help to prioritize risk and inform clinical decision-making. According to our review of the literature, a more rigorous evaluation of sleep variables may be warranted in such frameworks. Assessing sleep may reveal prognostic indicators for suicidal patients, inform clinical decision-making, and guide the development of more precise risk models for suicide. Delineating risk parameters related to sleep disturbances may also enhance assessment procedures, as well as prevention and intervention efforts. Importantly, sleep may be particularly amenable to treatment. Disturbed sleep, unlike many chronic risk factors, such as a history of suicide attempts (Joiner et al 2005), has the potential to be undone. As a warning sign, sleep may thus be a particularly useful factor, and a clinically meaningful intervention tool, to assess in the presence of suicidal ideation and depression.

Social rhythms may be an important target for future treatment research in this area. Irregular sleep and social rhythms both appear to serve as risk factors for future episodes of depression and mania (Leibenluft et al 1996; Ashman et al 1999). Moreover, treatments attempting to stabilize sleep and social rhythms appear to be effective in (1) improving medication treatment adherence and (2) reducing the likelihood of depression and mania relapse (Wehr et al 1998; Frank et al 2000). Sleep interventions may deserve further investigation in this regard. For example, it remains unclear whether sleep treatments and sleep psychoeducation may prospectively reduce risk for suicidality.

Several additional gaps in the sleep and suicide literature remain. Many investigations have evaluated suicidal behaviors and sleep symptoms, as well as the cross-sectional association between the two; however, we know of only one study which has formally examined suicidality among specific sleep disorders, such as sleep disordered breathing (Krakow et al 2000). Future research would do well to explore the prevalence of suicidal behaviors among those with other sleep disorders, such as sleep apnea, restless leg syndrome, primary insomnia, and circadian rhythm sleep disorders. Finally, an excellent review of the recent adolescent literature suggests that the association between sleep and suicidality remains an important yet neglected research area (Liu and Buysse 2006). Future studies are needed to examine sleep and suicidality in such populations.

\section{Conclusion}

In summary, evidence suggests that suicidal ideation and behaviors are closely associated with sleep disturbances, and in some cases, this association appears to exist above and beyond depression (Roberts et al 2001; Bernert et al 2005). Underlying neurobiological factors may, in part, account for this relationship. Suicide attempts and completed suicide are associated with serotonergic dysfunction, and serotonin release is furthermore involved in the regulation of both sleep and waking states. Circadian and homeostatic factors drive sleep, and preliminary evidence suggests that a 24-hour rhythm may exist for the timing of suicidal acts; nonetheless, these findings remain contradictory, and social factors in the timing of suicide and self-harm require further study. Nightmares show a unique association with suicide risk, whereas additional research is needed to clarify the association between insomnia and suicidality. Next, the prevalence of suicidality among specific sleep disorders, both before and after treatment, remains an understudied area. Finally, a study has yet to investigate whether sleep treatment or sleep psychoeducation reduces risk for suicidal behaviors. Sleep complaints may be particularly amenable to treatment. As a warning sign, disturbances in sleep may be especially useful to research and an important clinical target for suicide intervention efforts.

\section{Acknowledgments}

This work was supported, in part, by a grant from the National Institute of Mental Health to Rebecca A Bernert and Thomas Joiner (1 F31 MH080470-01) and by the John Simon Guggenheim Foundation.

\section{References}

Adrien J. 2002. Neurobiological bases for the relation between sleep and depression. Sleep Med Rev, 6:341-51.

Agargun MY, Beisoglu L. 2005. Sleep and suicidality: do sleep disturbances predict suicide risk? Sleep, 28:1039-40.

Agargun MY, Besiroglu L, Cilli AS, et al. 2007. Nightmares, suicide attempts, and melancholic features in patients with unipolar major depression. $J$ Affect Disord, 98:267-70.

Agargun MY, Cartwright R. 2003. REM sleep, dream variables and suicidality in depressed patients. Psychiatry Res, 119:33-9.

Agargun MY, Cilli AS, Kara H, et al. 1998. Repetitive and frightening dreams and suicidal behavior in patients with major depression. Compr Psychiatry, 39:198-202.

Agargun MY, Kara H, Solmaz M. 1997a. Sleep disturbances and suicidal behavior in patients with major depression. J Clin Psychiatry, 58:249-51.

Agargun MY, Kara H, Solmaz M. 1997b. Subjective sleep quality and suicidality in patients with major depression. $J$ Psychiatric Res, 31:377-81.

Asberg M. 1997. Neurotransmitters and suicidal behavior. The evidence from cerebrospinal fluid studies. Ann NY Acad Sci, 836:158-81.

Ashman SB, Monk TH, Kupfer DJ, et al. 1999. Relationship between social rhythms and mood in patients with rapid cycling bipolar disorder. Psychiatry Res, 86:1-8.

Bernert RA, Joiner TE, Cukrowicz KC, et al. 2005. Suicidality and sleep disturbances. Sleep, 28:1135-41.

Blenkiron P, House A, Milnes D. 2000. The timing of acts of deliberate self-harm: is there any relation with suicidal intent, mental disorder or psychiatric management?. J Psychosom Res, 49:3-6. 
Cartwright R, Young MA, Mercer P, et al. 1998. Role of REM sleep and dream variables in the prediction of remission from depression. Psychiatry Res, 80:249-55.

DeCoursey PJ. 2004. Overview of biological timing from unicells to humans. In: Dunlap JC, Loros JJ, DeCoursey PJ. eds. Chronobiology: biological timekeeping. Sunderland, Massachusetts: Sinauer Associates, Inc. p 20.

Dominguez RA, Goodnick PJ. 1995. Adverse events after the abrupt discontinuation of paroxetine. Pharmacotherapy, 15:778-80.

Doshi A, Boudreaux ED, Wang N, et al. 2005. National study of US emergency department visits for attempted suicide and self-inflicted injury, 1997-2001. Ann Emerg Med, 46:369-75.

Fawcett J, Scheftner WA, Fogg L. 1990. Time-related predictors of suicide in major affective disorder. Am J Psychiatry, 147:1189-94.

Frank E, Swartz HA, Kupfer DJ. 2000. Interpersonal and social rhythm therapy: managing the chaos of bipolar disorder. Biol Psychiatry, 48:593-604.

Fujino Y, Mizoue T, Tokui N, et al. 2005. Prospective cohort study of stress, life satisfaction, self-rated health, insomnia, and suicide death in Japan. Suicide Life Threat Behav, 35:227-37.

Gibbons RD, Hur K, Bhaumik DK, et al. 2005. The relationship between antidepressant medication use and rate of suicide. Arch Gen Psychiatry, 62:165-72.

Healy D, Whitaker C. 2003. Antidepressants and suicide: risk-benefit conundrums. J Psychiatry Neurosci, 28:331-7.

Joiner TE, Conwell Y, Fitzpatrick KK, et al. 2005. Four studies on how past and current suicidality related even when "everything but the kitchen sink" is covaried. J Abnorm Psychol, 114:291-303.

Joiner TE, Walker RL, Rudd MD, et al. 1999. Scientizing and routinizing the assessment of suicidality in outpatient practice. Prof Psychol Res $\operatorname{Pr}, 30: 447-53$.

Jouvet M. 1999. Sleep and serotonin: an unfinished story. Neuropsychopharmacology, 21:24s-27s.

Keshavan MS, Reynolds CF, Montrose D, et al. 1994. Sleep and suicidality in psychotic patients. Acta Psychiatr Scand, 89:122-5.

Krakow B, Artar A, Warner TD, et al. 2000. Sleep disorder, depression, and suicidality in female sexual assault survivors. Crisis, 21:163-70.

Leibenluft E, Albert PS, Rosenthal NE. 1996. Relationship between sleep and mood in patients with rapid-cycling bipolar disorder. Psychiatry Res, 63:161-8.

Leubkle JI, Greene RW, Semba K, et al. 1992. Serotonin hyperpolarizes cholinergic low-threshold burst neurons in the rat laterodorsal tegmental nucleus in vitro. Proc Natl Acad Sci USA, 89:743-7.

Lewinsohn PM, Rohde P, Seeley J. 1996. Adolescent suicidal ideation and attempts: prevalence risk factors, and clinical implications. Clin Psychol, 3:25-46.

Lin P, Tsai G. 2004. Association between serotonin transporter gene promoter polymorphism and suicide: results of a meta-analysis. Biol Psychiatry, 55:1023-30.

Liu X, Buysse DJ. 2006. Sleep and youth suicidal behavior: a neglected field. Curr Opin Psychiatry, 19:288-93.

Liu X. 2004. Sleep and adolescent suicidal behavior. Sleep, 27:1351-8.

Mann JJ, Brent DA, Arango V. 2001. The neurobiology and genetics of suicide and attempted suicide: a focus on the serotonergic system. Neuropsychopharmacology, 24:467-77.

Mann JJ. 2002. A current perspective of suicide and attempted suicide. Ann Intern Med, 136:302-11.
Maris RW. 2002. Suicide. Lancet, 360:319-26.

Mayers G, Baldwin DS. 2005. Antidepressants and their effect on sleep. Hum Psychopharmacol, 20:533-59.

Motahashi Y. 1990. Circadian variation in suicide attempts in Tokyo from 1978 to 1985. Suicide Life Threat Behav, 20:352-61.

Murphy SL. Deaths: final data for 1998. Natl Vital Stat Rep, 48:1-106.

National Mental Health Information Center. 2005. Suicide warning signs [online] Substance Abuse and Mental Health Services Administration (SAMHSA). Accessed 2 September 2006. URL: http://www. mentalhealth.samhsa.gov/publications/allpubs/walletcard/engwalletcard.asp

Pace-Schott EF, Gersh T, Silvestri R, et al. 2001. SSRI Treatment suppresses dream recall frequency but increases subjective dream intensity in normal subjects. J Sleep Res, 10:129-42.

Preti A, Miotto P. 2001. Diurnal variations in suicide by age and gender in Italy. J Affect Disord, 65:253-61.

Roberts RE, Roberts CR, Chen IG. 2001. Functioning of adolescents with symptoms of disturbed sleep. $J$ Youth Adolesc, 30:1-18.

Roman V, Walstra I, Luiten PG, et al. 2005. Too little sleep gradually desensitizes the serotonin 1A receptor system. Sleep, 28:1505-10.

Rowe JL, Bruce ML, Conwell Y. 2006. Correlates of suicide among home health care utilizers who died by suicide and community controls. Suicide Life Threat Behav, 36:65-75.

Sabo E, Reynolds CF, Kupfer DJ, et al. 1991. Sleep, depression, and suicide. Psychiatry Res, 36:265-77.

Saper CB, Chou TC, Scammell TE. 2001. The sleep switch: hypothalamic control of sleep and wakefulness. Trends Neurosci, 24:726-31.

Sharpley AL, Cowen PJ. 1995. Effect on pharmacological treatments on the sleep of depressed patients. Biol Psychiatry, 37:85-98.

Siegel JM. 1990. Mechanisms of sleep control. J Clin Neurophysiol, 7:49-65.

Smith MT, Perlis ML, Haythornthwaite JA. 2004. Suicidal ideation in outpatients with chronic muscoskeletal pain. Clin J Pain, 20:111-18.

Tanskanen A, Tuomilehto J, Viinamaki H, et al. 2001. Nightmares as predictors of suicide. Sleep, 24:844-7.

Trivedi MH, Rush AJ, Armitage R. 1999. Effects of fluoxetine on the polysomnogram in outpatients with major depression. Neuropsychopharmacology, 10:447-59.

Turvey CL, Conwell Y, Jones MP, et al. 2002. Risk factors for late-life suicide. Am J Geriatr Psychiatry, 10:398-406.

Ursin R. 2002. Serotonin and sleep. Sleep Med Rev, 6:57-69.

Van Houwelingen CAJ, Beersma DGM. 2001. Seasonal changes in 24-h patterns of suicide rates: a study on train suicides in The Netherlands. $J$ Affect Disord, 66:215-23.

Vogel G, Buffenstein A, Minter K, et al. 1990. Drug effects on REM sleep and endogenous depression. Neurosci Biobehav Rev, 14:49-63.

Warner CH, Bobo W, Warner C, et al. 2006. Antidepressant discontinuation syndrome. Am Fam Physician, 74:449-56.

Wehr TA, Turner EH, Shimada JM, et al. 1998. Treatment of a rapidly cycling bipolar patient by using extended bed rest and darkness to stabilize the timing and duration of sleep. Biol Psychiatry, 43:822-8.

Yerevanian BI, Koeck RJ, Feusner JD, et al. 2004. Antidepressants and suicidal behaviour in unipolar depression. Acta Psychiatr Scand, 110:452-8. 
